\title{
Hereditary Prostate Carcinoma
}

National Cancer Institute

\section{Source}

National Cancer Institute. Hereditary Prostate Carcinoma. NCI Thesaurus. Code

C103817.

Prostate carcinoma that has developed in relatives of patients with a history of prostate carcinoma. 